\title{
Baicalin ameliorates renal fibrosis via inhibition of transforming growth factor $\beta 1$ production and downstream signal transduction
}

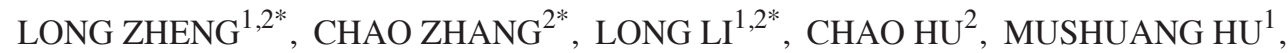 \\ NIYAZI SIDIKEJIANG ${ }^{1}$, XUANCHUAN WANG ${ }^{2}$, MIAO LIN ${ }^{2}$ and RUIMING RONG ${ }^{1,3}$ \\ ${ }^{1}$ Department of Urology; ${ }^{2}$ Shanghai Key Laboratory of Organ Transplantation; \\ ${ }^{3}$ Department of Blood Transfusion, Zhongshan Hospital, Fudan University, Shanghai 200032, P.R. China
}

Received December 22, 2015; Accepted December 19, 2016

DOI: $10.3892 / \mathrm{mmr} .2017 .6208$

\begin{abstract}
Previous studies have demonstrated the potential antifibrotic effects of baicalin in vitro, via examination of 21 compounds isolated from plants. However, its biological activity and underlying mechanisms of action in vivo remain to be elucidated. The present study aimed to evaluate the effect of baicalin on renal fibrosis in vivo, and the potential signaling pathways involved. A unilateral ureteral obstruction (UUO)-induced renal fibrosis model was established using Sprague-Dawley rats. Baicalin was administrated intraperitoneally every 2 days for 10 days. The degree of renal damage and fibrosis was investigated by histological assessment, and detection of fibronectin and collagen I mRNA expression levels. Epithelial-mesenchymal transition (EMT) markers, transforming growth factor- $\beta 1$ (TGF- $\beta 1$ ) levels and downstream phosphorylation of mothers against decapentaplegic 2/3 (Smad2/3) were examined in vivo and in an NRK-52E rat renal tubular cell line in vitro. Baicalin was demonstrated to markedly ameliorate renal fibrosis and suppress EMT, as evidenced by reduced interstitial collagen accumulation, decreased fibronectin and collagen I mRNA expression levels, upregulation of $\mathrm{N}$ - and E-cadherin expression levels, and downregulation of $\alpha$-smooth muscle actin and vimentin expression. Furthermore, baicalin decreased TGF- $\beta 1$ expression levels in serum and kidney tissue
\end{abstract}

Correspondence to: Dr Miao Lin, Shanghai Key Laboratory of Organ Transplantation, Zhongshan Hospital, Fudan University, 180 Fenglin Road, Shanghai 200032, P.R. China

E-mail: linmiaozs@sina.com

Professor Ruiming Rong, Department of Urology, Zhongshan Hospital, Fudan University, 180 Fenglin Road, Shanghai 200032, P.R. China

E-mail: rongruiming1969@163.com

${ }^{*}$ Contributed equally

Key words: renal fibrosis, baicalin, transforming growth factor- $\beta 1$, mothers against decapentaplegic 2/3, epithelial-mesenchymal transition following UUO, and suppressed Smad2/3 phosphorylation in rat kidney tissue. In vitro studies identified that baicalin may inhibit the phosphorylation of Smad2/3 under the same TGF- $\beta 1$ concentration. In conclusion, baicalin may protect against renal fibrosis, potentially via inhibition of TGF- $\beta 1$ production and its downstream signal transduction.

\section{Introduction}

Renal fibrosis is a common primary pathological feature of progressive kidney disease. Characteristics include hyperplasia of myofibroblasts and excessive deposition of the extracellular matrix (ECM) $(1,2)$. The ECM is primarily derived from myofibroblasts in kidneys, and excessive accumulation of ECM decreases the glomerular infiltration rate and renal function, eventually resulting in renal failure (3). Therefore, renal fibrosis is considered to be an irreversible process leading to end-stage renal failure. Previous studies have demonstrated that renal fibrosis is involved in numerous renal diseases, including chronic allograft nephropathy and membranous nephropathy $(4,5)$. Few strategies have been identified as effective for the prevention and treatment of renal fibrosis. Thus, there is a requirement for the development of novel therapies that prevent or ameliorate renal fibrosis.

Accumulating evidence indicates that in the event of renal injury, epithelial-mesenchymal transition (EMT) occurs, during which tubular epithelial cells lose their phenotypic markers [including neural (N)- and epithelial (E)-cadherin] and transform into fibroblasts, myofibroblasts or mesenchymal cells, gaining mesenchymal phenotypic markers, including vimentin and $\alpha$-smooth muscle actin (SMA) (6). Various factors are involved in this process, including cytokines, adhesion molecules and growth factors. Of these factors, transforming growth factor- $\beta 1$ (TGF- $\beta 1$ ) is considered an important regulator of EMT, as it causes renal scarring by activating the downstream mothers against decapentaplegic (Smad) signaling pathway. It has been demonstrated that the binding of TGF- $\beta 1$ to its receptor triggers the phosphorylation of Smad2 and 3, which combine with Smad4 to form the Smad complex. The complex is subsequently translocated to the nucleus to regulate target gene transcription, resulting in fibrogenesis $(3,7)$. Thus, inhibition of the production of 
TGF- $\beta 1$ or blockade of the TGF- $\beta 1$ signaling pathway is a potential strategy to prevent fibrogenesis.

Baicalin was originally isolated from the root of Scutellaria baicalensis, and is widely used for treating infectious and inflammatory diseases (8-10). Our previous study reported that this traditional Chinese herb root possesses protective effects against renal ischemia reperfusion injury via inhibition of the production of proinflammatory cytokines, including tumor necrosis factor (TNF)- $\alpha$ and interleukin (IL) $-1 \beta$ (11). A previous study demonstrated the antifibrotic effects of baicalin in rat livers via a reduction of profibrotic cytokines including serum TGF- $\beta 1$, TNF- $\alpha$ and IL- 6 , and an increase of the antifibrotic cytokine IL-10 (12). In addition, $\mathrm{Hu}$ et al (13) examined 21 compounds isolated from plants and demonstrated potential antifibrotic activities of baicalin in vitro; however, this requires further investigation in vivo. The underlying mechanisms of the antifibrotic activities of baicalin in vitro, and whether TGF- $\beta 1$ is involved in its effects on renal fibrosis, as has been demonstrated for hepatic fibrosis, remain to be determined.

The present study aimed to examine the antifibrotic effects of baicalin in a unilateral ureteral obstruction (UUO)-induced renal fibrosis rat model, and to investigate whether its underlying mechanisms involve the TGF- $\beta 1 / \mathrm{Smad}$ signaling pathway.

\section{Materials and methods}

Experimental animals. A total of 15 male Sprague-Dawley rats (weight, 200-250 g) were purchased from Shanghai SLAC Laboratory Animal Co., Ltd. (Shanghai, China). The rats were housed in specific pathogen-free grade conditions under a 12-h light/dark cycle with temperature and humidity ranging between $20-26^{\circ} \mathrm{C}$ and $40-70 \%$, respectively; free access to food and water was available for 1 week prior to the experiment. The present study was approved by the Care and Use of Laboratory Animals of the Ethical Commission of Fudan University (Shanghai, China) and performed under accepted ethical guidelines. Briefly, animals were acclimatized to laboratory conditions for 1 week prior to experimentation and anesthetized to minimize pain or discomfort during operation. Animals were sacrificed by pentobarbital overdose for tissue collection.

Unilateral renal fibrosis model. Rats were randomly allocated to three groups: Sham, UUO-induced fibrosis treated with normal saline and UUO-induced fibrosis treated with baicalin ( $\mathrm{n}=5 /$ group). Baicalin (catalog no. 572667; Sigma-Aldrich; Merck Millipore, Darmstadt, Germany) was diluted in normal saline to 20 and $100 \mathrm{mg} / \mathrm{kg}$ was administered by intraperitoneal injection every 2 days for 10 days. The UUO-induced fibrosis model was established as previously described (14). Pentobarbital $(40 \mathrm{mg} / \mathrm{kg}$, Shanghai USEN Biological Technology Co., Ltd., Shanghai, China) was used to anesthetize the rats. Following this, the abdominal cavity was exposed. The left ureter was identified, ligated at two points with non-absorbable 5-0 silk and cut between the ligatures. Rats in the sham group underwent ureter manipulation, but not ligation. Rats were placed on a $37^{\circ} \mathrm{C}$ heat pad during surgery to maintain body temperature.
After 10 days, rats were anesthetized with pentobarbital (40 mg/kg), and blood samples were collected from the heart and centrifuged at $2,000 \times \mathrm{g}$ for $10 \mathrm{~min}$ at $20^{\circ} \mathrm{C}$ to obtain serum samples. Following this, rats were sacrificed by overdose of pentobarbital. The kidneys were transversally cut at the midline and harvested.

Hematoxylin and eosin $(H \& E)$ staining. Paraffin-embedded rat kidney tissue was cut into 5-mm sections and subsequently deparaffinized and rehydrated prior to H\&E staining. The severity of renal interstitial injury was evaluated under light microscopy (magnification, x200). Sections from the sham, UUO and baicalin groups were evaluated according to a previous study performed by Lin et al (11). Tubular dilation, interstitial expansion and inflammatory cell infiltration were assessed using a tubulointerstitial injury scoring system as follows: 0 , normal; 1 , mild ( $\leq 25 \%) ; 2$, moderate ( $>25$ to $50 \%$ ); 3 , severe ( $>50 \%)$ (11). A total of 10 fields per specimen were randomly selected and assessed blindly by two examiners. Mean values of these estimates were used in the analyses.

Sirius red staining. Following deparaffinization and rehydration, paraffin-embedded kidney sections were incubated with $0.1 \%$ Sirius red solution at room temperature for $1 \mathrm{~h}$. The slides were subsequently washed twice in acidified water, dehydrated in absolute alcohol and cleared in xylene. A total of 10 fields per specimen were randomly selected and analyzed using Image-Pro Plus software version 6.0 (Media Cybernetics, Inc., Rockville, MD, USA) to evaluate the degree of renal fibrosis.

Immunohistochemical staining. Following deparaffinization and rehydration, paraffin-embedded kidney sections were blocked with $3 \%$ hydrogen peroxide and subsequently incubated for $1 \mathrm{~h}$ at $37^{\circ} \mathrm{C}$ with the following rabbit primary antibodies: Anti-E-cadherin (1:25; catalog no. ab15148; Abcam, Cambridge, UK), anti-N-cadherin (1:100; catalog no. 13116; Cell Signaling Technology, Inc. Danvers, MA, USA), anti- $\alpha$-SMA (1:200; catalog no. ab32575; Abcam, Cambridge, UK ) and anti-vimentin (1:100; catalog no. 5741; Cell Signaling Technology, Inc.). They were subsequently incubated with a horseradish peroxidase (HRP)-conjugated goat anti-rabbit secondary antibody (1:200; catalog no. ab6721; Abcam) for $1 \mathrm{~h}$ at room temperature. The reaction was visualized with a 3,3'-diaminobenzidine chromogen solution (Gene Tech Co., Ltd., Shanghai, China) and counterstained with hematoxylin. The slides were analyzed using Image-Pro Plus software version 6.0.

Western blot analysis. Renal tissue was homogenized in ice-cold radioimmunoprecipitation assay buffer (Vazyme Biotech Co., Ltd, Nanjing, China). Lysates were centrifuged at $12,000 \times \mathrm{g}$ at $4^{\circ} \mathrm{C}$ for $25 \mathrm{~min}$, following which the supernatant was collected and total proteins were quantified by bicinchoninic acid assay (Beyotime Institute of Biotechnology, Haimen, China). Samples $(20 \mu \mathrm{g})$ were separated via $10 \%$ sodium dodecyl sulfate-polyacrylamide gel electrophoresis (SDS-PAGE) and transferred onto polyvinylidene difluoride membranes at $310 \mathrm{~mA}$ for $110 \mathrm{~min}$. Membranes were blocked with $5 \%$ milk and subsequently incubated overnight at $4^{\circ} \mathrm{C}$ 
with the following rabbit primary antibodies: Anti-E-cadherin (1:500; Abcam), anti-N-cadherin $(1: 1,000)$, anti- $\alpha-S M A$ (1:1,000; catalog no. 14968; Cell Signaling Technology, Inc.), anti-vimentin (1:1,000), anti-GAPDH (1:1,000; catalog no. 2118), anti-phosphorylated (p)-Smad2 (1:1,000; catalog no. 3108), anti-p-Smad3 (catalog no. 9520) and anti-Smad2/3 (catalog no. 8685), all from Cell Signaling Technology, Inc., with gentle agitation. Membranes were washed with TBS-Tween-20 (1\%) and incubated with a HRP-conjugated goat anti-rabbit secondary antibody (1:5,000; catalog no. ab6721; Abcam) for $1 \mathrm{~h}$ at room temperature. Proteins were visualized using an Enhanced Chemiluminescence system (Thermo Fisher Scientific, Inc., Waltham, MA, USA). Band intensities were analyzed using Quantity-One 1-D Analysis software (version no. 4.62; Bio-Rad Laboratories, Inc., Hercules, CA, USA) and values were normalized against the value of GAPDH.

ELISA of serum TGF- $\beta 1$ expression levels. Serum TGF- $\beta 1$ levels were measured using an ELISA kit (catalog no. F16920; Shanghai Xitang Biotechnology Co., Ltd., Shanghai, China), according to the manufacturer's protocol.

Reverse transcription-quantitative polymerase chain reaction $(R T-q P C R)$. Total RNA was extracted from kidney tissue using TRIzol ${ }^{\circledR}$ reagent (Invitrogen; Thermo Fisher Scientific, Inc.). RNA (4 $\mu \mathrm{g})$ was reverse-transcribed using RevertAid First Strand cDNA Synthesis kit (Thermo Fisher Scientific, Inc.) into cDNA according to the manufacturer's protocol. qPCR was performed using a Mastercycler ${ }^{\circledR}$ ep realplex system (Eppendorf, Hamburg, Germany) in combination with the All-in-one ${ }^{\mathrm{TM}}$ qPCR Mix(GeneCopoeia, Rockville, MD, USA). Thermocycling conditions were as follows: Incubation for $2 \mathrm{~min}$ at $50^{\circ} \mathrm{C}$ and $10 \mathrm{~min}$ at $95^{\circ} \mathrm{C}$. This was followed by a 2-step PCR program of $95^{\circ} \mathrm{C}$ for $15 \mathrm{sec}$ and $60^{\circ} \mathrm{C}$ for $20 \mathrm{sec}$ for 45 cycles. Expression levels of target genes were normalized against GAPDH using the $2^{-\Delta \Delta C q}$ method (15). Gene-specific primers for rat TGF- $\beta 1$, fibronectin, collagen I and GAPDH are presented in Table I.

Cell culture and in vitro fibrosis model. The NRK-52E rat renal tubular epithelial cell line was purchased from the Shanghai Branch of the Chinese Academy of Science (Shanghai, China) and maintained in Dulbecco's modified Eagle's medium/F-12 nutrient mixture (DMEM/F-12; Shanghai BioSun Sci\&Tech Co., Ltd., Shanghai, China). A total of nine Petri dishes were seeded with NRK-52E cells at a density of $1 \times 10^{6}$ cells per dish. Cells were cultured with $10 \mu 1 \mathrm{DMEM} / \mathrm{F}-12$ containing $100 \mu \mathrm{l}$ PBS (control group), $100 \mu 1$ TGF- $\beta 1$ (3 ng/ml; Peprotech, Inc., Rocky Hill, NJ, USA; TGF- $\beta 1$ group) or $100 \mu 1$ TGF- $\beta 1+40 \mu 1$ baicalin (100 $\mu \mathrm{mol} / 1$; baicalin group; $\mathrm{n}=3$ /group). After 3 days, cells were collected for western blot analysis.

Statistical analysis. SPSS software version 19.0 (IBM SPSS, Armonk, NY, USA) was used for statistical analysis of the data by one-way analysis of variance, followed by a Bonferroni correction. Data are expressed as the mean \pm standard deviation. $\mathrm{P}<0.05$ was considered to indicate a statistically significant difference.
Table I. Primers used for reverse transcription-quantitative polymerase chain reaction.

\begin{tabular}{ll}
\hline Gene & \multicolumn{1}{c}{ Primer sequence $\left(5^{\prime}-3^{\prime}\right)$} \\
\hline GAPDH & F: AGGTCGGTGTGAACGGATTT \\
& R: GGGGTCGTTGATGGCAACA \\
Collagen I & F: GAGAGAGCATGACCGATGGA \\
& R: CGTGCTGTAGGTGAATCGAC \\
TGF- $\beta 1$ & F: CTTTGTACAACAGCACCCGC \\
& R: CGGGTGACTTCTTTGGCGTA \\
Fibronectin & F: TGGAGAGACAGGAGGAAATAGC \\
& R: CAGTGACAGCATACAGGGTGAT
\end{tabular}

F, forward; R, reverse; TGF- $\beta 1$, transforming growth factor- $\beta 1$.

\section{Results}

Baicalin attenuates UUO-induced renal fibrosis and injury. A total of 10 days following UUO, H\&E staining of obstructed kidneys was performed in all groups to assess the degree of renal injury and fibrosis. As presented in Fig. 1A, tubular structures had a healthy morphology and architecture in the sham group, whereas severe tubular dilation, interstitial expansion and inflammatory cell infiltration were observed in the UUO group. By contrast, baicalin treatment ameliorated tubulointerstitial lesions compared with the UUO group $(\mathrm{P}<0.001)$. Collagen accumulation was determined by Sirius red staining. Interstitial collagen accumulation in kidney tissue markedly increased following UUO $(\mathrm{P}<0.001)$. However, compared with the UUO group, baicalin significantly reduced the area of interstitial collagen deposition $(\mathrm{P}<0.001)$, although not to the level of the sham group $(\mathrm{P}<0.001)$. These results were further confirmed by tubular injury scores and the relative area of tubulointerstitial collagen deposition, as presented in Fig. 1B. Fibronectin and collagen I, components of ECM, were additionally analyzed to evaluate the degree of renal fibrosis. As presented in Fig. 1C, mRNA expression levels of the previously mentioned indicators were significantly elevated in kidney tissue following UUO compared with the sham group $(\mathrm{P}<0.001)$, whereas baicalin treatment markedly inhibited this effect $(\mathrm{P}<0.001)$, suggesting that baicalin inhibits the degree of renal injury and fibrosis in a UUO-induced fibrosis model.

Baicalin inhibits EMT in kidney tissue. EMT is a core process in the progression of renal fibrosis. The epithelial markers $\mathrm{N}$ - and E-cadherin and the mesenchymal markers $\alpha$-SMA and vimentin were examined to assess EMT (Fig. 2A). Immunohistochemical analysis revealed low baseline expression levels of vimentin and $\alpha$-SMA in the sham group, whereas expression levels of these proteins increased 10 days following UUO in the UUO group $(\mathrm{P}<0.001)$; by contrast, enhanced expression levels of $\mathrm{N}$ - and E-cadherin were observed in the sham group, which markedly decreased following UUO $(\mathrm{P}<0.001)$, indicating that renal fibrosis had occurred. However, compared with the UUO group, baicalin treatment significantly decreased the expression levels of 
A

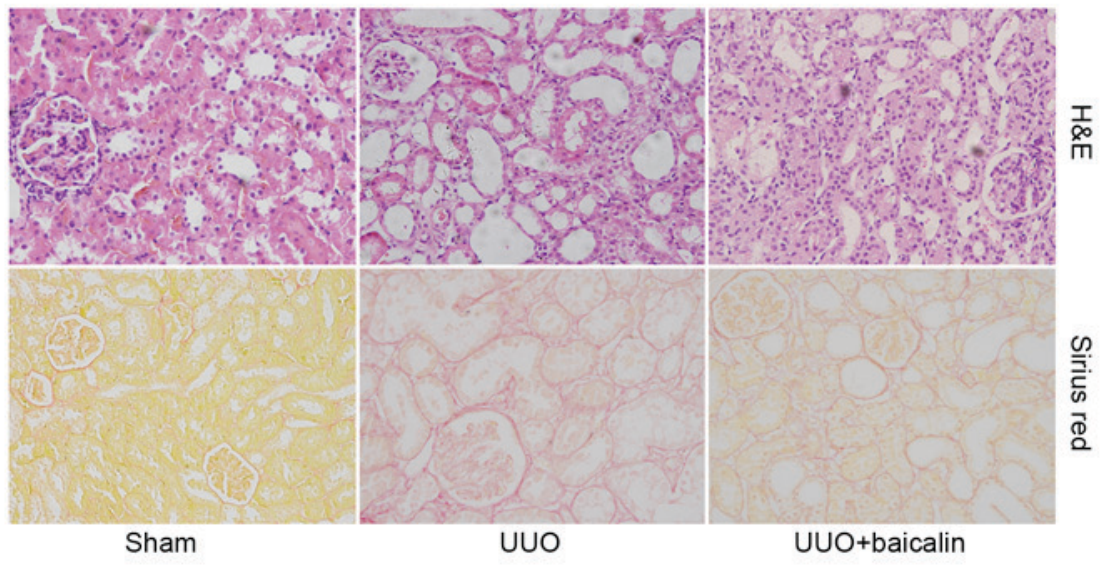

\section{$\underset{\substack{1 \\ \text { m }}}{\infty}$}

D:

B

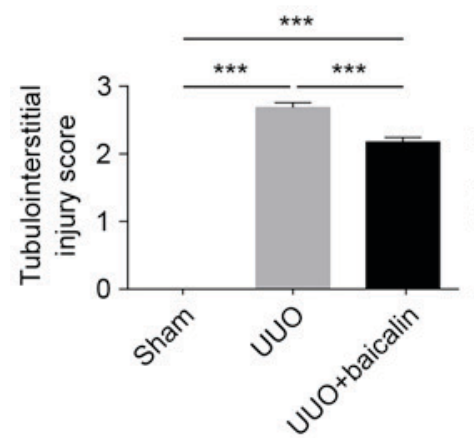

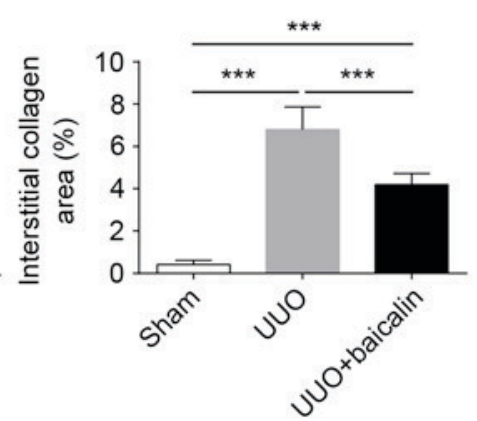

C

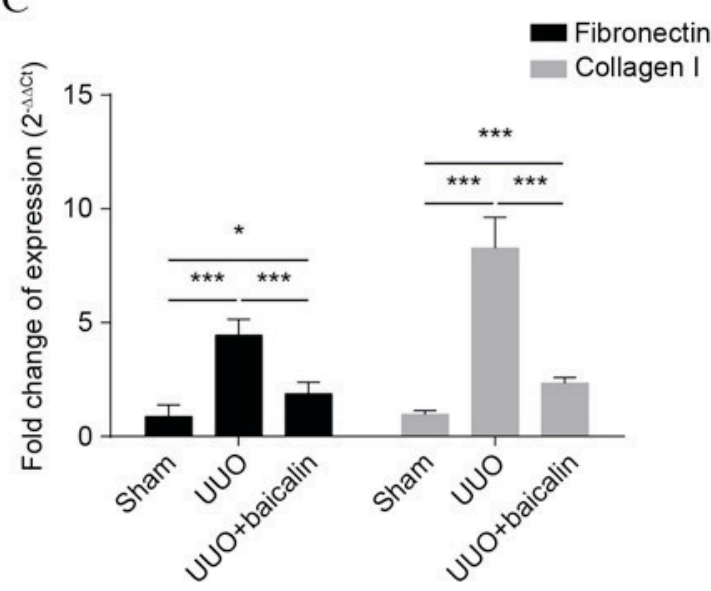

Figure 1. Baicalin attenuates UUO-induced renal injury and fibrosis. (A) Representative images of H\&E and Sirius red staining of kidney tissue in the sham, UUO and UUO + baicalin groups. (B) Quantification of tubular injury scores and tubulointerstitial collagen areas. (C) Fold-changes of mRNA expression levels of fibronectin and collagen I in the renal tissue of all groups. Data are expressed as the mean \pm standard deviation. ${ }^{*} \mathrm{P}<0.01$; ${ }^{* * *} \mathrm{P}<0.001$. UUO, unilateral ureteral obstruction; H\&E, hematoxylin and eosin.

$\alpha$-SMA and vimentin $(\mathrm{P}<0.001)$, and reversed the increased expression levels of $\mathrm{N}$ - and E-cadherin $(\mathrm{P}<0.001)$; however, not to the levels of the sham group (Fig. 2B). Similar results were obtained from western blot analysis (Fig. 3). These findings suggested that obstructive nephropathy following UUO was accompanied by the downregulation of $\mathrm{N}$ - and E-cadherin, and the upregulation of $\alpha$-SMA and vimentin. Furthermore, baicalin may partially reverse the alterations in the levels of these proteins, indicating that baicalin inhibits EMT in kidney tissue.

Baicalin treatment inhibits TGF- $\beta 1$ synthesis and phosphorylation of Smad2/3 in vivo. TGF- $\beta 1$ serves important roles in fibrogenesis. To identify whether TGF- $\beta 1$ is involved in the baicalin-mediated amelioration of renal fibrosis, TGF- $\beta 1$ levels were examined in serum and kidney tissue. As presented in Fig. $4 \mathrm{~A}$, and Table II, the levels of TGF- $\beta 1$ in serum following UUO $(0.592 \pm 0.042 \mathrm{ng} / \mathrm{ml})$ were significantly elevated compared with the sham group $(0.214 \pm 0.027 \mathrm{ng} / \mathrm{ml}$; $\mathrm{P}<0.001)$, whereas baicalin treatment markedly decreased these levels $(0.411 \pm 0.036 \mathrm{ng} / \mathrm{ml} ; \mathrm{P}<0.001)$, although they remained increased compared with the sham group $(\mathrm{P}<0.001)$.
Similarly, kidney TGF- $\beta 1$ mRNA expression levels were significantly increased in the UUO group compared with the sham group $(\mathrm{P}<0.001)$ (Fig. 4B). However, TGF- $\beta 1$ mRNA expression levels in the baicalin-treated group were significantly reduced compared with the UUO group $(\mathrm{P}<0.001)$. Therefore, baicalin treatment may reduce the production of TGF- $\beta 1$ in UUO-induced fibrogenesis.

To investigate if the TGF- $\beta 1$ signaling pathway was attenuated by baicalin treatment, phosphorylation of Smad 2 and 3 was examined by western blot analysis (Fig. 4C). Phosphorylation of Smad2 and 3 was almost undetectable in the sham group, and significantly increased 10 days following UUO $(\mathrm{P}<0.001)$. Notably, although increased compared with the sham group $(\mathrm{P}<0.05)$, phosphorylation of these proteins in the baicalin-treated group was significantly reduced compared with the UUO group $(\mathrm{P}<0.01)$ (Fig. 4D). These results indicated that 10 days of ureteral obstruction triggered the phosphorylation of Smad2 and 3, which was markedly attenuated by baicalin.

Baicalin inhibits TGF- $\beta 1$ signaling in an in vitro TGF- $\beta 1$-induced fibrosis model. To investigate whether the 
Table II. Serum transforming growth factor- $\beta 1$ expression levels.

\begin{tabular}{|c|c|c|c|c|}
\hline Group & Value & Mean & Standard deviation & P-value \\
\hline 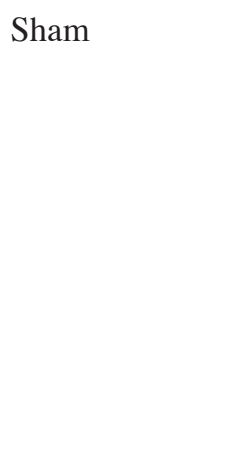 & $\begin{array}{l}0.233 \\
0.229 \\
0.212 \\
0.206 \\
0.251 \\
0.257 \\
0.183 \\
0.181 \\
0.194 \\
0.197\end{array}$ & 0.214 & 0.027 & $\begin{array}{c}<0.001 \text { (vs. UUO) } \\
<0.001 \text { (vs. UUO + baicalin) }\end{array}$ \\
\hline 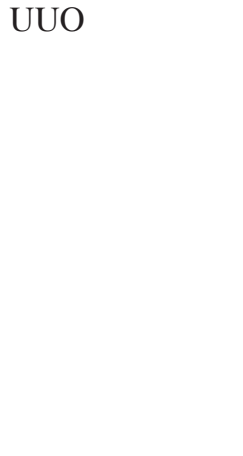 & $\begin{array}{l}0.567 \\
0.571 \\
0.621 \\
0.616 \\
0.575 \\
0.573 \\
0.657 \\
0.653 \\
0.544 \\
0.541\end{array}$ & 0.592 & 0.042 & $\begin{array}{c}<0.001 \text { (vs. sham) } \\
<0.001 \text { (vs. UUO + baicalin) }\end{array}$ \\
\hline UUO + baicalin & $\begin{array}{l}0.435 \\
0.441 \\
0.419 \\
0.426 \\
0.373 \\
0.378 \\
0.453 \\
0.448 \\
0.362 \\
0.372\end{array}$ & 0.411 & 0.036 & $\begin{array}{l}<0.001 \text { (vs. sham) } \\
<0.001 \text { (vs. UUO) }\end{array}$ \\
\hline
\end{tabular}

UUO, unilateral ureteral obstruction.

phosphorylation of Smad2/3 may be decreased by baicalin in the presence of TGF- $\beta 1$, the phosphorylation of Smad2/3 was examined by western blot analysis in an NRK-52E rat renal tubular epithelial cell line (Fig. 5A). Similar to the results in vivo, the phosphorylation of $\mathrm{Smad} 2 / 3$ was significantly increased in the TGF- $\beta 1$ group compared with the control group $(\mathrm{P}<0.001$ and $\mathrm{P}<0.01$, respectively; Fig. 5B). Notably, baicalin treatment was demonstrated to significantly decrease Smad2/3 phosphorylation $(\mathrm{P}<0.01$ and $\mathrm{P}<0.05$, respectively), although the levels of phosphorylation remained increased compared with the control group, further demonstrating that baicalin treatment may ameliorate renal fibrosis. To demonstrate the effect of the inhibition of phosphorylation of Smad2/3, protein expression levels of EMT markers in NRK-52E cells were additionally assessed by western blot analysis (Fig. 5C). Similar to the results in vivo, baicalin treatment inhibited the TGF- $\beta 1$-induced decrease of $\mathrm{N}$ - and E-cadherin expression levels $(\mathrm{P}<0.01$ and $\mathrm{P}<0.001$, respectively), and inhibited the increase of vimentin and $\alpha$-SMA induced by TGF- $\beta 1(\mathrm{P}<0.001$ and $\mathrm{P}<0.05$, respectively; Fig. 5D). This indicated that baicalin treatment decreases the phosphorylation of Smad2/3 in NRK-52E cells even in the presence of TGF- $\beta 1$, thereby inhibiting the process of EMT.

\section{Discussion}

Various treatments and therapeutic targets with the aim of ameliorating renal fibrosis have previously been evaluated, including drugs, endocrine hormones, the complement system and microRNAs $(2,5,16-18)$. However, inactivation of a specific hormone may affect the secretion of other hormones, inhibition of complement may impair immune function and antagonists of microRNAs are not as stable as compounds and only function for a short period of time, which limits the clinical application of these methods. Therefore, safe and efficient strategies to prevent renal fibrosis are required. A previous study demonstrated the low cytotoxicity of baicalin, which indicates the safety of baicalin (13). Additionally, 
A

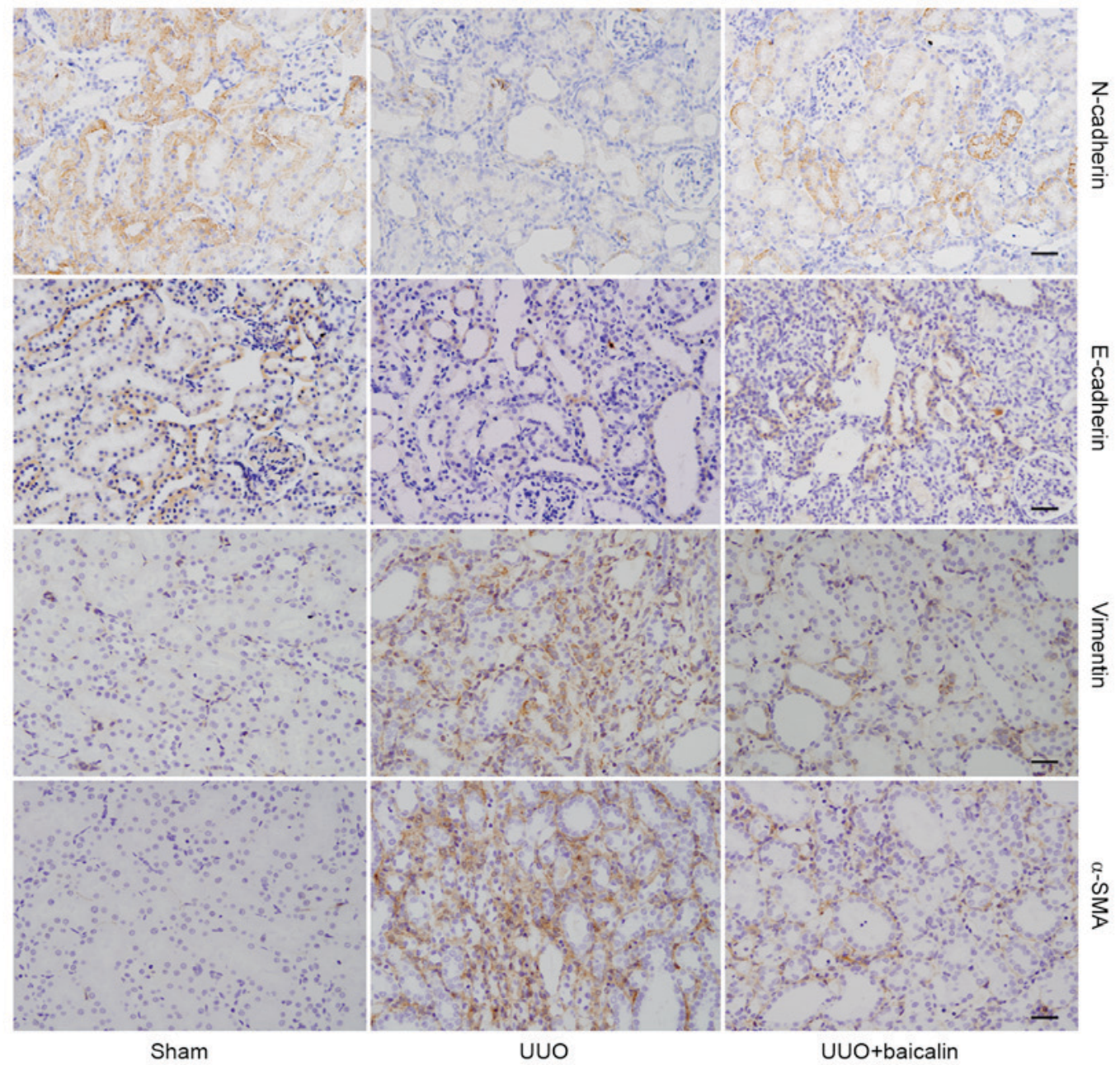

B
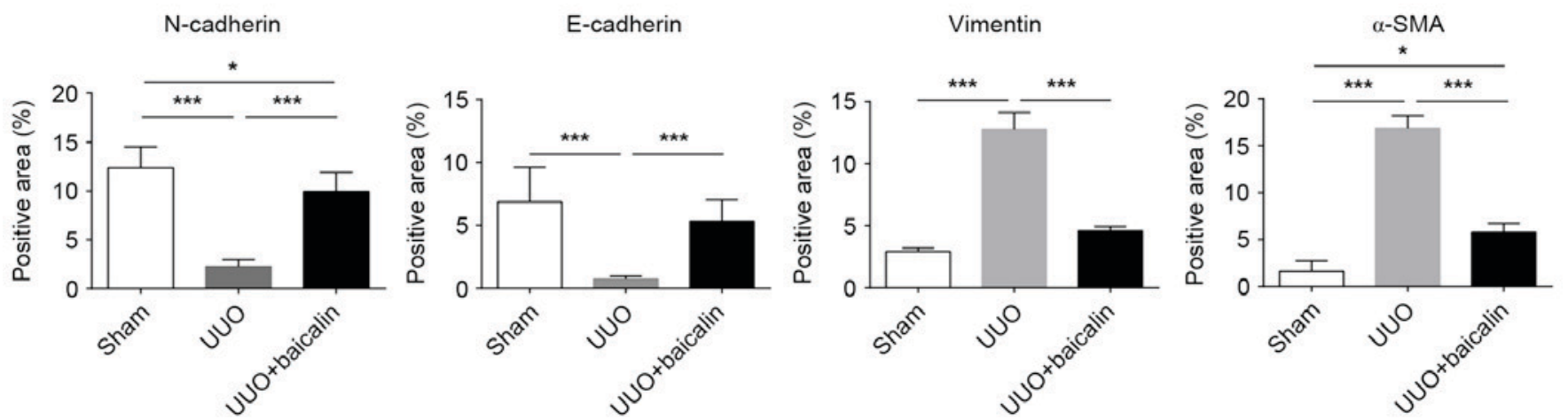

Figure 2. Baicalin treatment inhibits epithelial-mesenchymal transition in kidney tissue, as assessed by immunohistochemistry. (A) Representative images of $\mathrm{N}$ - and E-cadherin, vimentin, and $\alpha$-SMA staining in kidney sections from sham, UUO and UUO + baicalin groups. (B) Percentages of positive areas of $\mathrm{N}$ - and E-cadherin, vimentin and $\alpha$-SMA. Data are expressed as the mean \pm standard deviation. ${ }^{*} \mathrm{P}<0.05$; ${ }^{* * * *} \mathrm{P}<0.001$. UUO, unilateral ureteral obstruction; $\mathrm{N}$, neural; E, epithelial; $\alpha$-SMA, $\alpha$-smooth muscle actin.

another previous study reported that baicalin treatment may ameliorate the effects of fibrosis within various organs. Jia et al (19) demonstrated that baicalin attenuated bleomycin-induced lung fibrosis via downregulation of connective tissue growth factor. Liu et al (20) revealed that baicalin inhibited the synthesis of collagen I in pulmonary arteries, and attenuated pulmonary vascular remodeling via upregulation of a disintegrin and metalloproteinase with thrombospondin motifs 1 expression. Qiao et al (21) demonstrated that the combination of mesenchymal stem cells and baicalin exhibited a more effective therapeutic effect in hepatic fibrosis compared with treatment with mesenchymal stem cells alone. Yang et al (22) demonstrated that the active components of Yang-Gan-Wan, rosmarinic acid and baicalin 
A
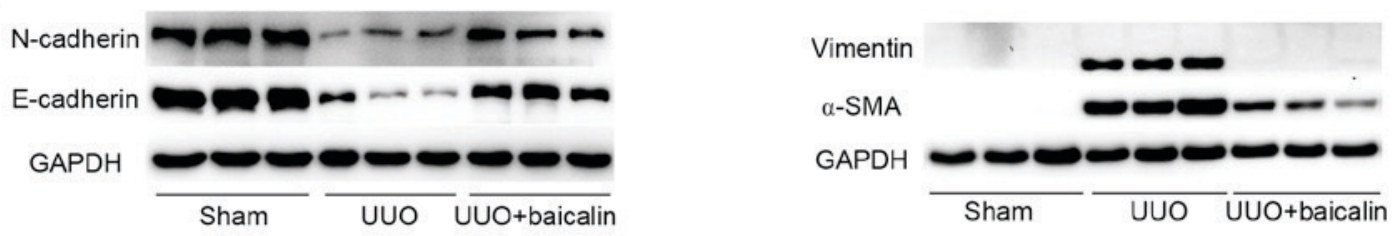

B
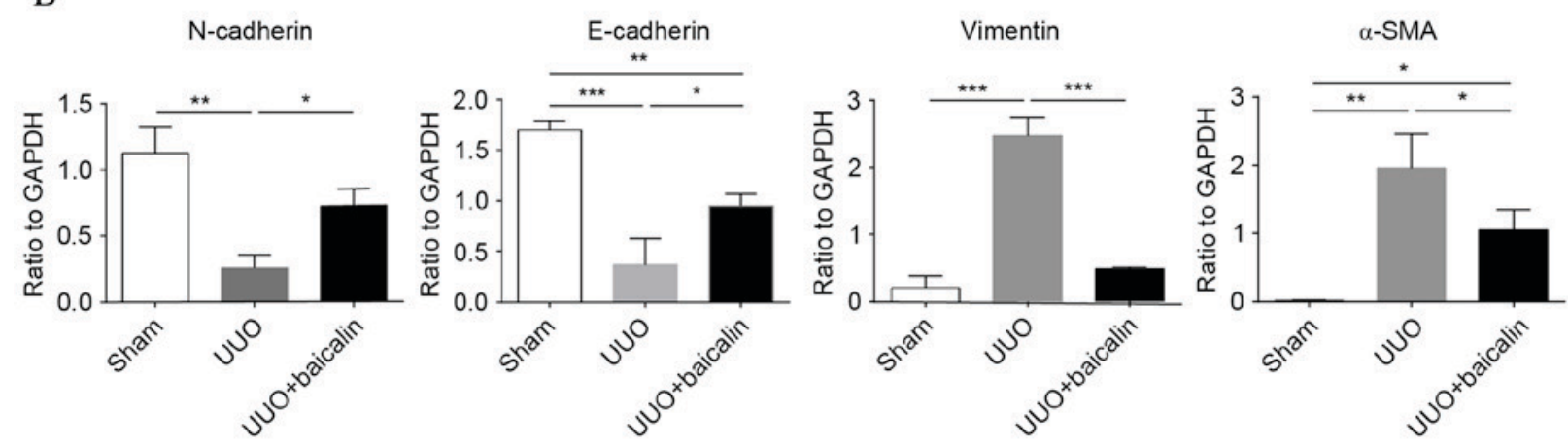

Figure 3. Baicalin treatment inhibits epithelial-mesenchymal transition in kidney tissue, as assessed by western blotting. (A) Representative western blot images demonstrating the expression of $\mathrm{N}$ - and E-cadherin, vimentin, and $\alpha$-SMA in kidney tissue from the sham, UUO and UUO + baicalin groups. (B) The ratio of $\mathrm{N}$ - and E-cadherin, vimentin, and $\alpha$-SMA expression levels to GAPDH. Data are expressed as the mean \pm standard deviation. ${ }^{*} \mathrm{P}<0.05$; ${ }^{* *} \mathrm{P}<0.01$; ${ }^{* * *} \mathrm{P}<0.001$. UUO, unilateral ureteral obstruction; $\alpha$-SMA, $\alpha$-smooth muscle actin; N, neural; E, epithelial.

had an antifibrotic effect on liver fibrosis via inhibition of peroxisome proliferator-activated receptor- $\gamma$. In the present study, baicalin was systematically demonstrated to have a protective effect against renal fibrosis by reducing interstitial collagen accumulation, decreasing mRNA expression levels of fibronectin and collagen $\mathrm{I}$, upregulating $\mathrm{N}$ - and $\mathrm{E}$-cadherin expression levels, and downregulating $\alpha$-SMA and vimentin expression levels. In addition, baicalin was identified to ameliorate renal fibrosis via inhibition of TGF- $\beta 1$ production and its downstream signal transduction. A schematic of the potential effects of baicalin on the TGF- $\beta 1$ signaling pathway is presented in Fig. 6. Even in the presence of TGF- $\beta 1$, baicalin reduced the phosphorylation of $\mathrm{Smad} 2 / 3$ in vitro.

The majority of chronic kidney diseases (CKDs) are characterized by excessive ECM accumulation in the interstitium and glomerulus, disrupting healthy kidney structure, decreasing the glomerular infiltration rate and eventually resulting in renal failure (3). The UUO-induced renal fibrosis model has been frequently used to mimic the pathological alterations of chronic obstructive nephropathy that are commonly observed in patients with CKD (23). The present study demonstrated that baicalin markedly protected against renal damage, reduced the area of interstitial collagen deposition, and suppressed the accumulation of the ECM components fibronectin and collagen I, demonstrating its protective effect against renal fibrosis.

EMT, defined by the loss of epithelial characteristics and the acquisition of a mesenchymal phenotype, has been hypothesized to be a primary underlying mechanism of ECM and fibrogenesis (24). Myofibroblasts are regarded as the effector cells of renal fibrosis (3). During EMT, myofibroblast derivation occurs within the epithelium. The transformation of renal tubular epithelial cells to myofibroblasts results in the excessive production and accumulation of ECM and eventually renal fibrogenesis $(25,26)$. A previous study demonstrated that in the absence of EMT, the accumulation of myofibroblasts was reduced and reversed (27), revealing the crucial role of EMT in fibrogenesis. In the present study, following UUO, baicalin was demonstrated to exert a protective effect against the process of EMT, as evidenced by upregulation of the epithelial markers $\mathrm{N}$ - and E-cadherin, and downregulation of the mesenchymal markers $\alpha$-SMA and vimentin.

TGF- $\beta 1$, the most abundant subtype of the TGF- $\beta$ family, has been extensively studied as a mediator of fibrogenesis (28). Accumulating evidence has demonstrated that TGF- $\beta 1$ serves a central role in the pathogenesis of renal fibrosis in experimental models and human kidney diseases (3). Various underlying mechanisms have been reported, including induction of ECM production via Smad-dependent canonical and non-canonical signaling pathways, inhibition of ECM degradation via suppression/inhibition of matrix metalloproteinases, and induction of the transdifferentiation of renal tubular cells to myofibroblasts by EMT (3). Furthermore, Ding and Choi (29) reported that autophagy, which is additionally regulated by TGF- $\beta$, was involved in renal fibrosis. In the present study, the reduction of serum and kidney tissue TGF- $\beta 1$ expression levels by baicalin treatment implied that baicalin affects the expression levels of this pro-fibrotic cytokine.

The TGF- $\beta 1 /$ Smad signaling pathway is regarded as a crucial mediator of fibrogenesis (3). In the present study, 
A

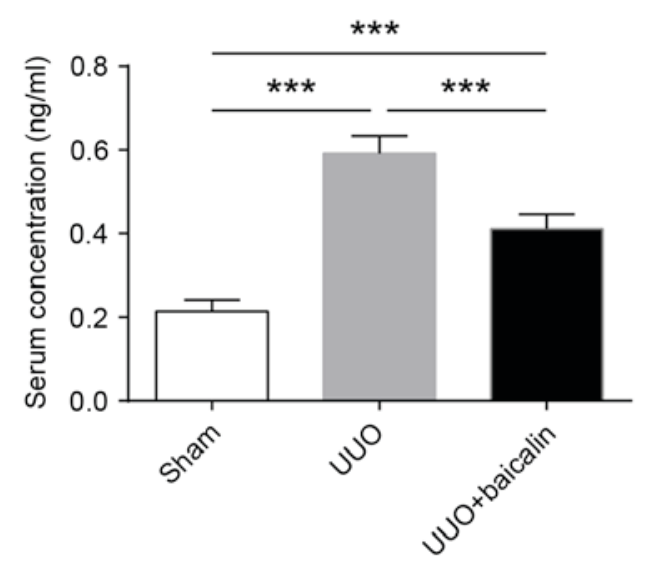

B

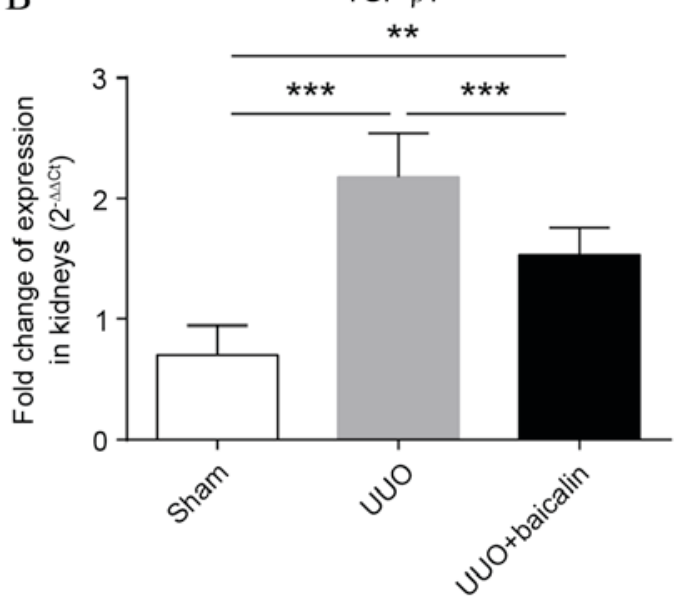

$\mathrm{C}$

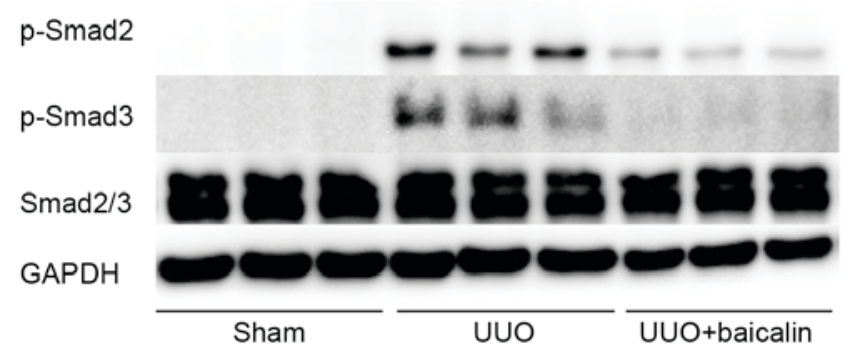

$\mathrm{D}$
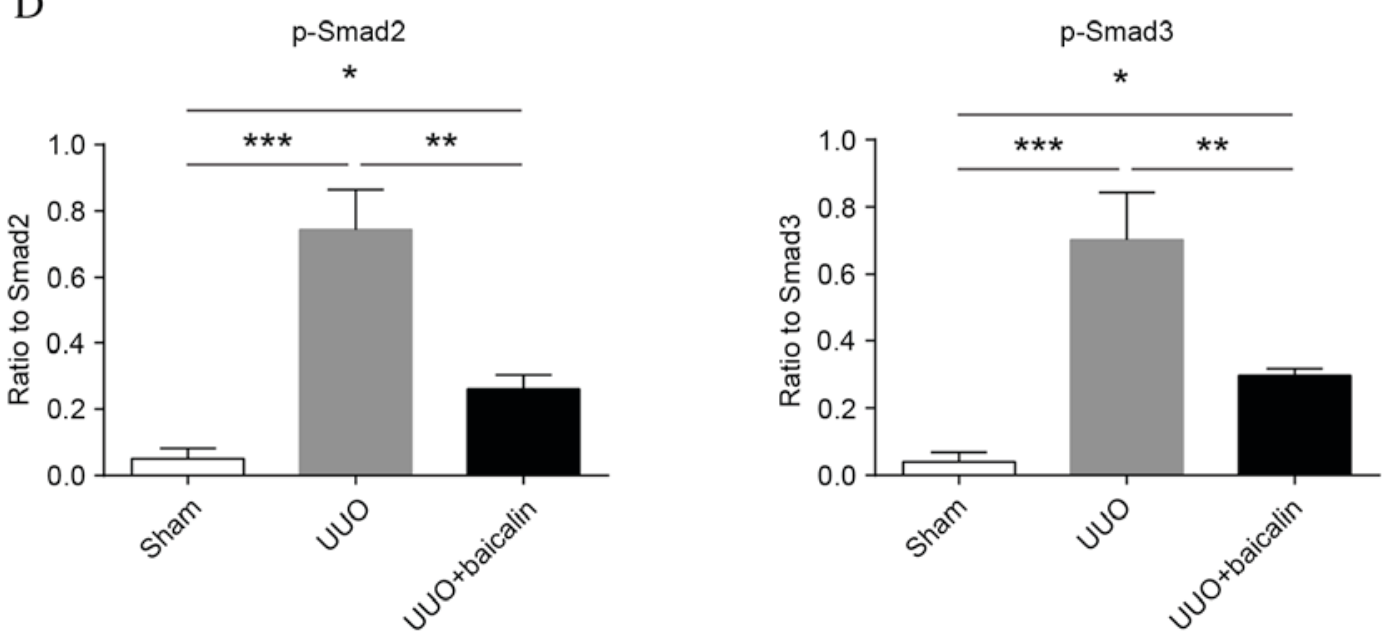

Figure 4. Baicalin treatment inhibits TGF- $\beta 1$ expression levels and phosphorylation of Smad2/3 in vivo. (A) Quantification of serum TGF- $\beta 1$ levels in the sham, UUO and UUO + baicalin groups, as measured by ELISA. (B) Fold-change of mRNA expression levels of TGF- $\beta 1$ in renal tissue. (C) Representative western blot images of p-Smad2, p-Smad3 and Smad2/3 expression in sham, UUO and UUO + baicalin groups. GAPDH served as an internal control. (D) The ratio of $\mathrm{p}-\mathrm{Smad} 2 / 3$ to Smad2/3. Data are expressed as the mean \pm standard deviation. ${ }^{*} \mathrm{P}<0.05 ;{ }^{* *} \mathrm{P}<0.01 ;{ }^{* * *} \mathrm{P}<0.001$. UUO, unilateral ureteral obstruction; TGF- $\beta 1$, transforming growth factor- $\beta 1 ; \mathrm{p}$, phosphorylated; Smad, mothers against decapentaplegic.

the significantly enhanced phosphorylation of Smad2/3 in the UUO group compared with the sham group, and the decreased phosphorylation of Smad2/3 in the baicalin group compared with the UUO group in vivo, revealed the potential participation of the TGF- $\beta 1 /$ Smad signaling pathway in the amelioration of renal fibrosis by baicalin. To further investigate whether the phosphorylation of Smad2/3 may be decreased by baicalin in the presence of TGF- $\beta 1$, the effect of baicalin on the phosphorylation of Smad2/3 in NRK-52E cells was examined under controlled 
A

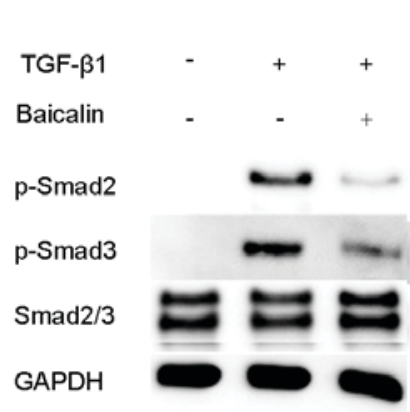

B

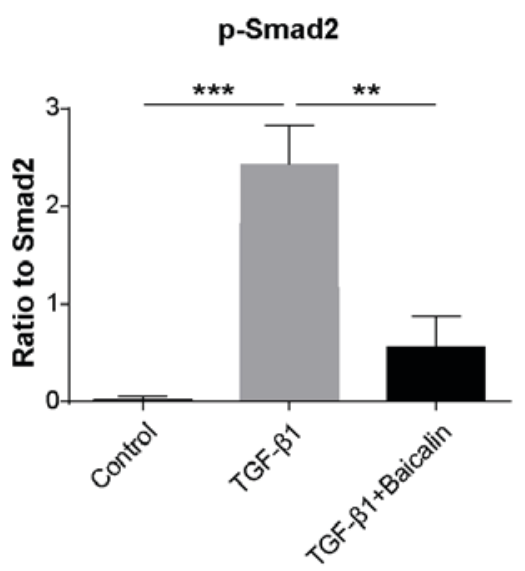

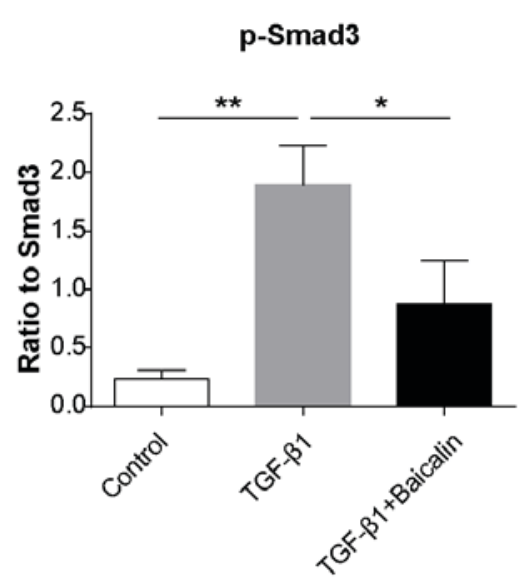

$\mathrm{C}$

$\begin{array}{lllll}\text { TGF- } \beta 1 & - & + & + \\ \text { Baicalin } & - & - & + \\ \mathrm{N} \text {-cadherin } & & & + \\ \text { E-cadherin } & - & - \\ \text { GAPDH }\end{array}$

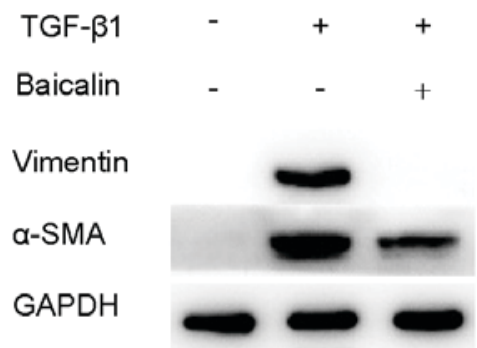

$\mathrm{D}$

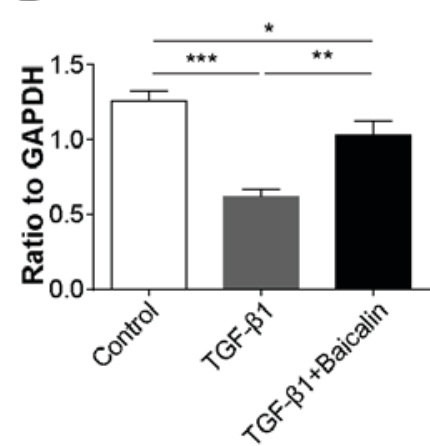

E-cadherin

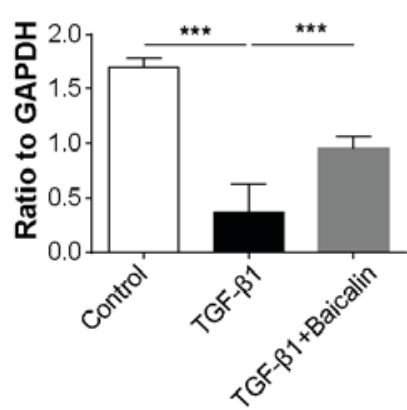

Vimentin

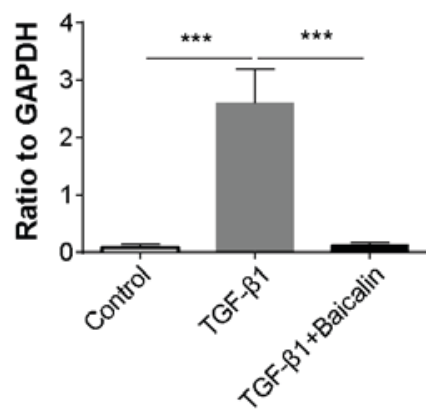

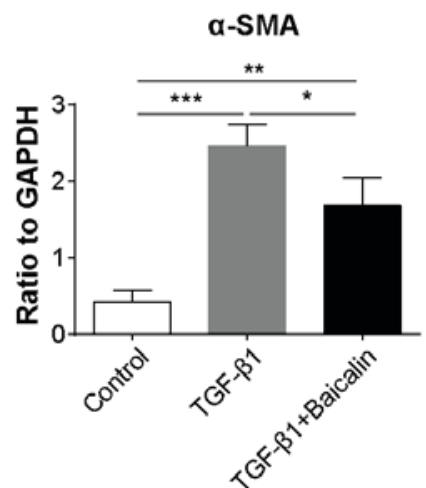

Figure 5. Baicalin treatment inhibits TGF- $\beta 1$ signaling in a NRK-52E rat renal tubular epithelial cell line in an in vitro TGF- $\beta 1$-induced fibrosis model. (A) Representative western blot images of $\mathrm{p}-\mathrm{Smad} 2, \mathrm{p}-\mathrm{Smad} 3$ and Smad2/3 expression in control, TGF- $\beta 1$ and TGF- $\beta 1+$ baicalin groups. GAPDH served as an internal control. (B) The ratio of p-Smad2/3 to Smad2/3 expression levels. (C) Representative western blot images of N- and E-cadherin, vimentin and $\alpha$-SMA expression. (D) Quantification of the ratio of $\mathrm{N}$ and E-cadherin, vimentin, and $\alpha$-SMA expression levels to GAPDH. Data are expressed as the mean \pm standard deviation. "P $<0.05 ;{ }^{* *} \mathrm{P}<0.01 ;{ }^{* * *} \mathrm{P}<0.001$. $\alpha$-SMA, $\alpha$-smooth muscle actin; N, neural; E, epithelial; p, phosphorylated; Smad, mothers against decapentaplegic; $\alpha$-SMA, $\alpha$ smooth muscle actin; TGF- $\beta 1$, transforming growth factor- $\beta 1$.

concentrations of TGF- $\beta 1$ in vitro. In comparison with the TGF- $\beta 1$ group, baicalin inhibited the phosphorylation of Smad2/3. Furthermore, phosphorylation of Smad2/3 reversed the process of EMT even in the presence of TGF- $\beta 1$, demonstrating that baicalin may ameliorate renal fibrosis even when TGF- $\beta 1$ is expressed. However, whether baicalin ameliorates renal fibrosis by decreasing the binding of TGF- $\beta 1$ to its receptors, or by directly inhibiting the phosphorylation of Smad2/3 in vitro, remains unknown and requires further investigation. In conclusion, the present study demonstrated that baicalin may ameliorate renal fibrosis in vivo, via reduction of TGF- $\beta 1$ and its downstream signal transduction, and in the presence of TGF- $\beta 1$ in vitro. This suggested that baicalin may be a potential novel therapeutic agent for the clinical prevention of renal fibrosis.

\section{Acknowledgements}

The present study was supported by the Shanghai Health and Family Planning Commission (grant no. 2014JQ008A) and the National Natural Science Foundation of China (grant no. 81500568). 


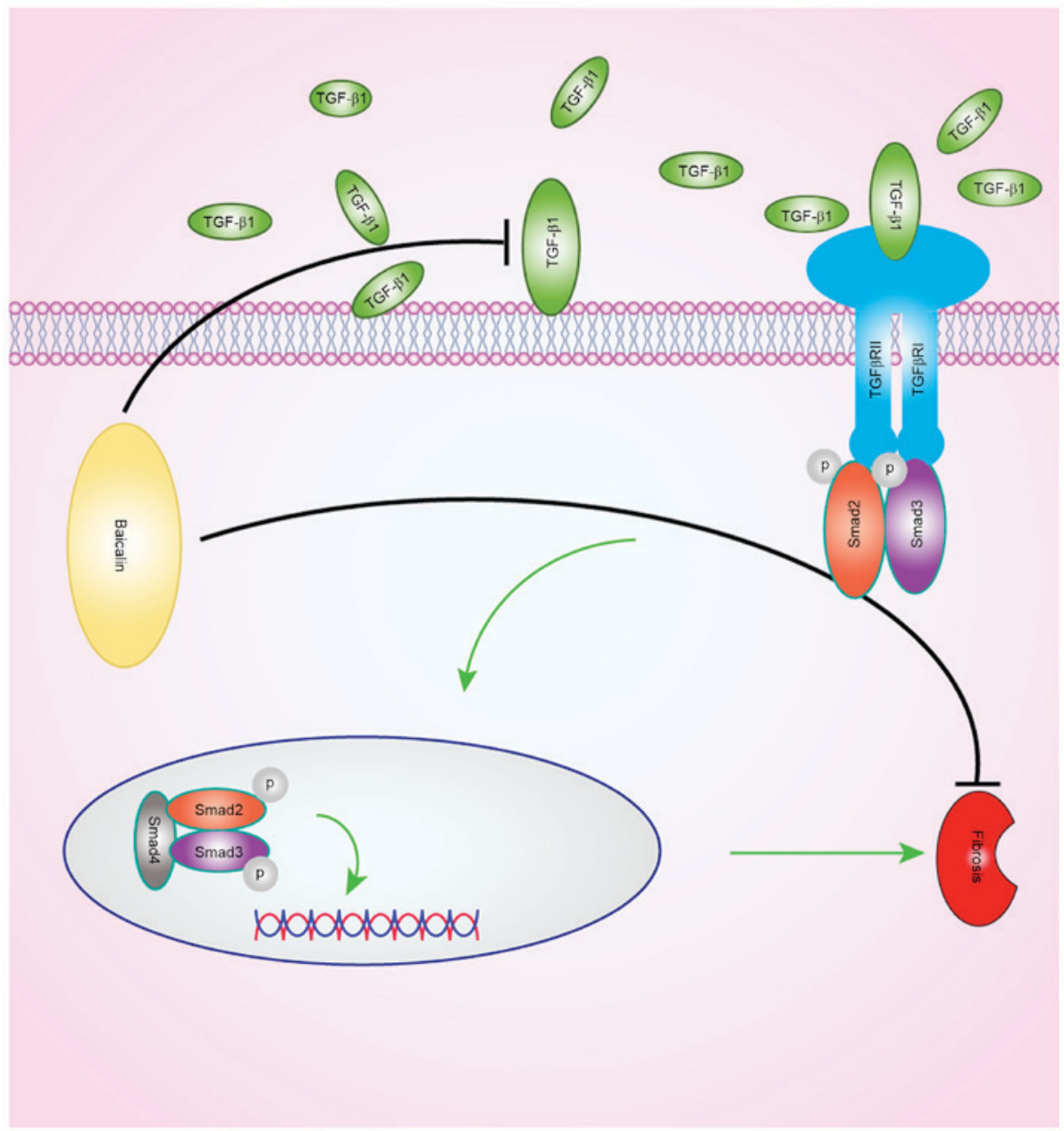

Figure 6. Schematic diagram of the potential mechanisms of action of baicalin on the amelioration of renal fibrosis. The binding of TGF- $\beta 1$ to its receptor triggers Smad2/3 phosphorylation. Once combined with Smad4, the Smad complex is formed, which later translocates to the nucleus to regulate target gene transcription, resulting in fibrogenesis. Baicalin ameliorates renal fibrosis via inhibition of TGF- $\beta 1$ production and its downstream signal transduction. Smad, small mothers against decapentaplegic; TGF- $\beta 1$, transforming growth factor $\beta 1 ;$ p, phosphorylated.

\section{References}

1. Wynn TA: Common and unique mechanisms regulate fibrosis in various fibroproliferative diseases. J Clin Invest 117: 524-529, 2007.

2. Guo Y, Li Z, Ding R, Li H, Zhang L, Yuan W and Wang Y: Parathyroid hormone induces epithelial-to-mesenchymal transition via the $\mathrm{Wnt} / \beta$-catenin signaling pathway in human renal proximal tubular cells. Int J Clin Exp Pathol 7: 5978-5987, 2014.

3. Lan HY: Diverse roles of TGF- $\beta$ /Smads in renal fibrosis and inflammation. Int J Biol Sci 7: 1056-1067, 2011.

4. Becker LE, Weritz B, Yi X, Gross-Weissmann ML, Waldherr R, Zeier $\mathrm{M}$ and Sommerer C: Evolution of allograft fibrosis and function in kidney transplant recipients: A retrospective analysis of stable patients under CNI and mTORi. Transpl Int 28: 553-564, 2015.

5. Li TT, Zhang XH, Jing JF, Li X, Yang XQ, Zhu FH, Tang W and Zuo JP: Artemisinin analogue SM934 ameliorates the proteinuria and renal fibrosis in rat experimental membranous nephropathy. Acta Pharmacol Sin 36: 188-199, 2015.

6. Lan HY: Tubular epithelial-myofibroblast transdifferentiation mechanisms in proximal tubule cells. Curr Opin Nephrol Hypertens 12: 25-29, 2003.

7. Nakao A, Imamura T, Souchelnytskyi S, Kawabata M, Ishisaki A, Oeda E, Tamaki K, Hanai J, Heldin CH, Miyazono K and ten Dijke P: TGF-beta receptor-mediated signalling through Smad2, Smad3 and Smad4. EMBO J 16: 5353-5362, 1997.

8. Cao Y, Mao X, Sun C, Zheng P, Gao J, Wang X, Min D, Sun H, Xie N and Cai J: Baicalin attenuates global cerebral ischemia/reperfusion injury in gerbils via anti-oxidative and anti-apoptotic pathways. Brain Res Bull 85: 396-402, 2011
9. Tang YJ, Zhou FW, Luo ZQ, Li XZ, Yan HM, Wang MJ, Huang FR and Yue SJ: Multiple therapeutic effects of adjunctive baicalin therapy in experimental bacterial meningitis. Inflammation 33: 180-188, 2010.

10. Zhu J, Wang J, Sheng Y, Zou Y, Bo L, Wang F, Lou J, Fan X, Bao R, Wu Y, et al: Baicalin improves survival in a murine model of polymicrobial sepsis via suppressing inflammatory response and lymphocyte apoptosis. PLoS One 7: e35523, 2012.

11. Lin M, Li L, Li L, Pokhrel G, Qi G, Rong R and Zhu T: The protective effect of baicalin against renal ischemia-reperfusion injury through inhibition of inflammation and apoptosis. BMC Complement Altern Med 14: 19, 2014.

12. Peng XD, Dai LL, Huang CQ, He CM and Chen LJ: Correlation between anti-fibrotic effect of baicalin and serum cytokines in rat hepatic fibrosis. World J Gastroenterol 15: 4720-4725, 2009.

13. Hu Q, Noor M, Wong YF, Hylands PJ, Simmonds MS, Xu Q, Jiang D, Hendry BM and Xu Q: In vitro anti-fibrotic activities of herbal compounds and herbs. Nephrol Dial Transplant 24: 3033-3041, 2009

14. Satoh M, Kashihara N, Yamasaki Y, Maruyama K, Okamoto K, Maeshima Y, Sugiyama H, Sugaya T, Murakami K and Makino H: Renal interstitial fibrosis is reduced in angiotensin II type 1a receptor-deficient mice. J Am Soc Nephrol 12: 317-325, 2001.

15. Livak KJ and Schmittgen TD: Analysis of relative gene expression data using real-time quantitative PCR and the 2(-Delta Delta $\mathrm{C}(\mathrm{T})$ ) method. Methods 25: 402-408, 2001

16. Wang DT, Huang RH, Cheng X, Zhang ZH, Yang YJ and Lin X Tanshinone IIA attenuates renal fibrosis and inflammation via altering expression of TGF- $\beta /$ Smad and $N F-\kappa B$ signaling pathway in 5/6 nephrectomized rats. Int Immunopharmacol 26: 4-12, 2015 . 
17. Li L, Chen L, Zang J, Tang X, Liu Y, Zhang J, Bai L, Yin Q, $\mathrm{Lu} \mathrm{Y}$, Cheng J, et al: C3a and C5a receptor antagonists ameliorate endothelial-myofibroblast transition via the $\mathrm{Wnt} / \beta$-catenin signaling pathway in diabetic kidney disease. Metabolism 64: 597-610, 2015.

18. Denby L, Ramdas V, Lu R, Conway BR, Grant JS, Dickinson B, Aurora AB, McClure JD, Kipgen D, Delles C, et al: MicroRNA-214 antagonism protects against renal fibrosis. J Am Soc Nephrol 25: 65-80, 2014.

19. Jia H, Chen XL, Chen C, Hu YY and Yun XJ: Baicalin prevents the up-regulation of connective tissue growth factor in fibrotic lungs of rats. Sheng Li Xue Bao 62: 535-540, 2010 (In Chinese).

20. Liu P, Yan S, Chen M, Chen A, Yao D, Xu X, Cai X, Wang L and Huang X: Effects of baicalin on collagen I and collagen III expression in pulmonary arteries of rats with hypoxic pulmonary hypertension. Int J Mol Med 35: 901-908, 2015.

21. Qiao H, Tong Y, Han H, Xu W, Ren Z, Ouyang J and Chen Y: A novel therapeutic regimen for hepatic fibrosis using the combination of mesenchymal stem cells and baicalin. Pharmazie 66: 37-43, 2011.

22. Yang MD, Chiang YM, Higashiyama R, Asahina K, Mann DA, Mann J, Wang CC and Tsukamoto H: Rosmarinic acid and baicalin epigenetically derepress peroxisomal proliferator-activated receptor $\gamma$ in hepatic stellate cells for their antifibrotic effect. Hepatology 55: 1271-1281, 2012.
23. Wang W, Zhou PH, Xu CG, Zhou XJ, Hu W and Zhang J: Baicalein attenuates renal fibrosis by inhibiting inflammation via down-regulating NF- $\kappa \mathrm{B}$ and MAPK signal pathways. J Mol Histol 46: 283-290, 2015.

24. Masszi A and Kapus A: Smaddening complexity: The role of Smad3 in epithelial-myofibroblast transition. Cells Tissues Organs 193: 41-52, 2011.

25. O'Connor JW and Gomez EW: Cell adhesion and shape regulate TGF-betal-induced epithelial-myofibroblast transition via MRTF-A signaling. PLoS One 8: e83188, 2013.

26. Zeisberg M and Kalluri R: The role of epithelial-to-mesenchymal transition in renal fibrosis. J Mol Med (Berl) 82: 175-181, 2004

27. Yang J, Shultz RW, Mars WM, Wegner RE, Li Y, Dai C, Nejak K and Liu Y: Disruption of tissue-type plasminogen activator gene in mice reduces renal interstitial fibrosis in obstructive nephropathy. J Clin Invest 110: 1525-1538, 2002.

28. Lebrin F, Deckers M, Bertolino P and Ten DP: TGF-beta receptor function in the endothelium. Cardiovasc Res 65: 599-608, 2005.

29. Ding Y and Choi ME: Regulation of autophagy by TGF- $\beta$ Emerging role in kidney fibrosis. Semin Nephrol 34: 62-71, 2014. 